\title{
Ezrin, Radixin and Moesin: Minor Molecule with Major Impact:
} A Review

\author{
Dr. Deshant Agarwal ${ }^{1}$, Dr. Manas Bajpai ${ }^{2}$, Dr. S.S. Vanaki ${ }^{3}$, Dr. R.S. Puranik ${ }^{4}$ \\ ${ }^{I}$ (Department of Oral and Maxillofacial Pathology, NIMS Dental College, Jaipur, India) \\ ${ }^{2}$ (Department of Oral and Maxillofacial Pathology, NIMS Dental College, Jaipur, India) \\ ${ }_{3}^{3}$ (Department of Oral and Maxillofacial Pathology, PMNM Dental College, Bagalkot, India) \\ ${ }^{4}$ (Department of Oral and Maxillofacial Pathology, PMNM Dental College, Bagalkot, India)
}

\begin{abstract}
ERM (ezrin/radixin/moesin) proteins, concentrated in actin rich cell-surface structures, cross-link actin filaments with the plasma membrane. Recent work using genetic approaches has revealed a surprising wealth of phenotypes and functions for ERMs in the context of both normal tissues and disease states. Such a wide array of phenotypes, from loss of epithelial integrity to disruption of embryonic anterior-posterior polarity, seems to suggest a wide range of molecular functions. They are involved in the formation of microvilli, cell-cell adhesion, maintenance of cell shape, cell motility, membrane trafficking and metastasis. The functions of ERM proteins are regulated by their conformational changes: the intramolecular interaction between the $\mathrm{N}$ and C-terminal domains of ERM proteins masks several binding sites, leading to a dormant protein. Different activation signals regulate ERM proteins functions by modulating these intramolecular interactions.
\end{abstract}

Key words: Ezrin, Radixin, Moesin, Cytoskeleton, Membrane Dynamics, Metastasis

\section{Introduction}

ERM proteins (Ezrin/Radixin/Moesin) are structural linkers between the plasma membrane and the actin cytoskeleton, located mainly just beneath the plasma membrane of cellular protrusions such as microvilli. Recent work using genetic approaches has revealed a surprising wealth of phenotypes and functions for ERMs in the context of both normal tissues and disease states. Such a wide array of phenotypes, from loss of epithelial integrity to disruption of embryonic anterior-posterior polarity, seems to suggest a wide range of molecular functions. Now, it appears that they are also signal transducers. Here, we review the studies on ERM, with the emphasis on its function in physiological and pathological processes.

\section{History of the ERM family:}

Ezrin was the first protein identified in many systems and the best studied of the ERM family. It is a protein of 585 amino acids with an isoelectric point of 6.15 and a theoretical molecular mass of $69 \mathrm{kDa}$. This polypeptide was called ezrin in recognition of Ezra Cornell University where it was purified. ${ }^{[1]}$

Radixin, a 583 -amino acid polypeptide shares $75.3 \%$ identity with the human ezrin..$^{[1]}$

Moesin (membrane-organizing extension spike protein) is a 577 -amino acid polypeptide which shares $71.7 \%$ sequence identity with the mouse ezrin. ${ }^{[2]}$

\section{Structural insights into ERM function.}

ERMs are characterized by the presence of an 300 amino acid plasma membrane-associated FERM domain in the amino terminus, followed by a long region with a high $\alpha$-helical propensity and terminating in a carboxy-terminal domain, known as the C-terminal ERM-association domain (C-ERMAD), that has the ability to bind the FERM domain or filamentous actin (F-actin). ERM function is conformationally regulated by head to tail folding (that is, binding of the C-terminal C-ERMAD to the N-terminal FERM domain). All ERMs exist in an apparently dormant, closed conformation and that release of the C-ERMAD from the FERM domain is necessary for their full activation and exposes binding sites in the FERM domain and the F-actin-binding site of the C-ERMAD. Current ideas indicate that the dormant protein is spring-loaded, so that when the affinity between the FERM domain and the C-ERMAD is reduced; the molecule pops open, allowing it to connect the membrane to the underlying actin cytoskeleton. ${ }^{[3,4]}$

\section{Activation of ERMs by phosphorylation:}

In vivo studies in Drosophila melanogaster, ${ }^{[5]}$ have provided evidences that ERMs are activated when they adopt an open conformation following phosphorylation of the regulatory Thr. Additionally Cyclindependent kinase 5 (CDK5) can also phosphorylate Thr235 of ezrin ${ }^{[6]}$, which lies on the FERM-C-ERMAD interface directly opposite Thr567 ${ }^{[7]}$. In addition to Thr residues, ezrin can be phosphorylated on Tyr 145 and 
Tyr353 by various Tyr kinases, including epidermal growth factor receptor (EGFR) ${ }^{[8]}$, but how these modifications affect the conformation and function of ezrin is still unclear.

\section{Binding partners of ERMs.}

As mentioned above, phosphorylation of ERMs at the regulatory Thr residue reduces the affinity of the C-ERMAD for the FERM domain, and this active conformation allows other proteins including CD44, CD43 and ICAM2 ${ }^{[9]}$ to bind. A distinct site on the FERM domain can bind to the related scaffolding proteins ERMbinding phosphoprotein 50 (EBp50; also known as NHERF1) and NHE3 kinase A regulatory protein (E3KARp; also known as NHERF2). Therefore, the FERM domain can bind at least two different classes of membraneassociated proteins. EBp50 and E3KARp bind multiple different membrane proteins themselves ${ }^{[10]}$, so there are several proteins that have the potential to bind the FERM domain directly or indirectly.

\section{Inactivation of ERM proteins}

The activation of ERM is largely studied; little is known about their inactivation. It has been shown that ezrin is degraded by calpain, a protease regulated by intracellular calcium. The degradation of ezrin leads to a disruption of the actin cytoskeleton. However, moesin and radixin are resistant to calpain ${ }^{[11]}$. In their nonphosphorylated state ERM proteins are present as monomers and have no site available for interaction with other molecules due to the intramolecular interaction. Thus, one possible mechanism of inactivation is the dephosphorylation of the activated ERM.

\section{Localization of the ERM proteins}

The localization of the ERM proteins has been analyzed in various tissues and culture cell lines. Although ezrin, radixin and moesin are co-expressed in most cultured cells, they exhibit a tissue-specific expression pattern in the whole body ${ }^{[12]}$.

Ezrin is highly concentrated in intestine, stomach, lung and kidney although moesin is prominent in lung and spleen, and radixin in liver and intestine ${ }^{[13,14]}$. Ezrin is expressed in epithelial and mesothelial cells while moesin is expressed in endothelial cells ${ }^{[12]}$. The brush border of intestinal epithelial cells express only ezrin, and hepatocytes express only radixin ${ }^{[15]}$. At the subcellular level, the three ERM are concentrated in actin-rich surface structures such as microvilli, filopodia and membrane ruffles ${ }^{[16,17]}$. The localization of radixin is slightly distinct from ezrin and moesin since it was originally identified as a component of adherent junctions. It is also localized at focal contacts, in the cleavage furrow and the contractile ring of cultured cells ${ }^{[13,14]}$. However, these localizations are controversial ${ }^{[18]}$.

\section{ERM: Functional insight:}

\subsection{Formation of cell surface structures:}

Several observations suggested that ERM proteins were involved in membrane dynamics ${ }^{[19,20,21]}$. More recently, several functional analyses demonstrated that they are involved in membrane structures formation. When thymoma cells are cultured in presence of antisense oligonucleotides, which suppress the expression of the three ERM, microvilli disappear from the cell surface ${ }^{[22]}$. A treatment of primary retinal pigment epithelium (PRE) culture with ezrin antisense oligonucleotides caused complete disappearance of apical microvilli ${ }^{[23]}$.

\subsection{Cell-substrate and cell-cell adhesion}

The suppression of ERM expression by antisense oligonucleotides in thymoma cells as well as in mouse epithelial cells leads to the destruction of both cell-cell and cell-substrate adhesion ${ }^{[22]}$. Overexpression of ezrin in insect cells enhances cell adhesion ${ }^{[24]}$.

\subsection{Maintenance of cell shape and cell motility}

Ezrin plays an important role in pseudopodial extension in Fos-transformed Rat-1 fibroblasts, and maintains cell shape in normal Rat-1 cells.

\subsection{Membrane trafficking:}

Recent data reveal that ERM are also involved in membrane trafficking. Cao et al. ${ }^{[25]}$ (1999) have shown that EBP50, ERM proteins and the actin cytoskeleton have a specialized function in the endocytic sorting of a subset of membrane proteins. The proper sorting of internalized $\beta 2$-adrenergic receptors requires EBP50 to interact with the cytoplasmic tail of the $\beta 2$-adrenergic receptor and with ERM ${ }^{[26]}$.

\subsection{ERM and embryonic development:}

Before the eight-cell stage, embryonic cells are undifferentiated. The polarization of the cells, which occurs during the eight-cell stage, at compaction, is an essential step for the epithelial differentiation that is 
achieved $24 \mathrm{~h}$ later. Before compaction, ezrin is present all around the cell cortex and at the cell-cell contacts. As soon as the cells polarize, ezrin localizes to the apical pole of the blastomeres, and is found exclusively in microvilli ${ }^{[27]}$.

\subsection{Oocyte polarity:}

The ability to asymmetrically orient and localize the cytoskeleton is known to be essential to the stable maintenance of cell polarity. Although ERMs organize the cortical cytoskeleton asymmetrically and thereby promote adherens junction stability and epi thelial integrity, they do not seem to be directly involved in establishing apical-basal polarity in epithelial cells. However, partial depletion of Moesin from the $D$. melanogaster oocyte can result in severe anterior- posterior polarity defects owing to the mislocalization of gene products that specify the posterior end, including oskar mRNA and Staufen ${ }^{[28,29]}$.

\subsection{Mitosis:}

Recent studies have shown that in D. melanogaster Moesin functions in enhancing cortical stiffness to promote cell rounding during mitosis ${ }^{[30,31,32]}$. In $D$. melanogaster cultured cells, reduction in Moesin levels using RNA interference results in multiple defects during mitosis, including cell shape abnormalities and delay in anaphase onset ${ }^{(33)}$. Measurements using an atomic force microscope indicate that cortical rigidity fails to increase during mitosis when Moesin is depleted ${ }^{[30]}$.

\subsection{Epithelial morphogenesis and integrity:}

Moesin act as as a binding partner for Bitesize, a cytoplasmic protein, is recruited to the apical membrane domain through interactions with the apical polarity complex. In the absence of Bitesize function, early aspects of apical-basal polarity, including the formation of adherens junctions, occur normally but, subsequently, epithelial organization and the adherens junctions break down ${ }^{[34,35]}$. Loss of moesin function display a disruption in overall morphology and epithelial integrity ${ }^{[36,37]}$. Few studies suggested that bitesize interaction with Moesin is specific to the early stages of epithelial morphogenesis, or that it has redundancy with other, currently unknown proteins. ${ }^{[38]}$ Similarly, although some Moesin-mutant imaginal epithelial cells lose cell integrity and polarity, many remain integrated into the epithelial layer ${ }^{[36]}$, suggesting that ERMs are not absolutely required for adherens junction stability in established epithelia.

\subsection{Lumen morphogenesis:}

Studies in both $C$. elegans and mice have recently suggested that ERM-1 has an important function at the apical-junctional interface during lumen morphogenesis ${ }^{[39,40]}$. Loss of ERM-1 led to the appearance of cyst-like structures along the lumen in these epithelia, accompanied by constrictions, twisting and occlusions of intestinal lumen as well as abnormal positioning of adherens junctions, suggesting a defect in lumen formation. ${ }^{[39,40,41]}$

\subsection{Metastasis:}

Many studies document increased ezrin expression and/or activity specifically in metastatic tumours, with human and mouse osteosarcomas and rhabdomyosarcomas being the best-studied examples ${ }^{[42,43]}$. Ezrin is upregulated early during metastatic progression and later as established metastases expand; it is downregulated, helps in survival of metastatic nodules. This suggests a role for ezrin in tumour invasion, initially from the primary tumour and subsequently during metastatic expansion. This is consistent with studies implicating ERMs in junctional remodelling and/or stability - processes known to be defective in tumour invasion ${ }^{[44]}$. Several ezrin-associated transmembrane proteins, such as CD44, podoplanin and podocalyxin, have also been implicated independently in tumour metastasis ${ }^{[45]}$. Interestingly ERMs have been proposed to have both positive and negative roles in lymphocyte migration ${ }^{[46,47]}$.

\subsection{The immunological synapse - an example of ERM functional diversity.}

Unstimulated $\mathrm{T}$ cells are spherically uniform and covered in microvilli, so to form the synapse the microvilli have to be locally disassembled at the site of interaction, which is achieved through ERM dephosphorylation ${ }^{[48]}$. In addition, bulky glycoproteins (such as CD43) have to be moved away, and the T cell receptor has to be recruited to the site of the synapse. Adhesion molecules have to then hold the two cells together. ERMs have been implicated in all these processes ${ }^{[49,50,51]}$. Interestingly, a similar array of ERMmediated cytoskeletal rearrangements occurs during infection of lymphocytes by HIV-1.

\subsection{The role of ERMs in retroviral and bacterial infections:}

ERMs have a surprisingly complex role in cells infected by retroviruses or bacteria. Infection of lymphocytes by HIV-1 induces a complex array of cortical rearrangements that lead to the formation of what has been termed the virological synapse ${ }^{[52]}$ by analogy to the immunological synapse. On contact, GP120 on the surface of the virus binds CD4 on the lymphocyte surface, recruiting this transmembrane protein and the 
associated protein CXC-chemokine receptor 4 (CXCR4) to the contact region. Concomitantly, there is a local activation and accumulation of moesin and ezrin together with filamentous actin (F-actin), the actin-binding protein filamin and the severing protein cofilin to the synapse. Small interfering RNA-mediated knockdown of ERMs strongly diminishes the ability of HIV-1 to enter and infect cells ${ }^{[3,54]}$ and prevents the redistribution of CD4 and CXCR4 into the synapse ${ }^{[53]}$. CD4-CXCR4 clustering depends on the actin cytoskeleton, suggesting that ERMs facilitate virological synapse formation by anchoring actin filaments.

A recent study has revealed a fascinating mechanism that bacteria use to circumvent the host's leukocyte response ${ }^{[55]}$. Endothelial cells respond to the adhering bacterial colony in a similar manner to leukocytes by accumulating transmembrane proteins (ICAM1, ICAM2, CD44 and E-selectin), F-actin and active ERMs to the region of contact. As a result, infected endothelial cells are unable to respond properly to leukocytes because the $N$. meningitides colony titrates away a limited pool of available ERMs and blocks the formation of a leukocyte docking site, preventing leukocyte adhesion.

\section{Conclusion and perspectives}

In conclusion, ERM proteins are complex proteins in terms of their structure and functions: posttranslational modifications and association with various regulators modulate their intramolecular interactions between the carboxy- and amino-terminal domains and control their activities as cross-linkers and signal transducers. There are hints that ERMs may regulate cortical cytoskeletal organization in multiple ways. Its ability to interact with multiple partners, possibly in a combinatorial manner, provides the potential for considerable functional diversity. Thus, further elucidation of ERM binding partners, and the contexts in which they interact, will be a key area for future research. Another important and still unexplored area of ERM research is how they are regulated since we know little about the mechanisms upstream these events. Integration of this information into a comprehensive model of regulated ERM-mediated membrane complex formation will, in turn, increase our understanding of many complex biological and disease processes.

\section{REFERENCES}

[1] Funayama, N., Nagafuchi, A., Sato, N., Tsukita, S., (1991). Radixin is a novel member of the band 4.1 family. J. Cell Biol. 115, 1039-1048.

[2] Sato, N., Funayama, N., Nagafuchi, A., Yonemura, S., Tsukita, S., (1992). A gene family consisting of ezrin, radixin and moesin. Its specific localization at actin filament/plasma membrane association sites. J. Cell Sci. 103, 131-143.

[3] Turunen, O., Wahlstrom, T. \& Vaheri, A. (1994) Ezrin has a COOH-terminal actin-binding site that is conserved in the ezrin protein family. J. Cell Biol. 126, 1445-1453.

[4] Gary, R. \& Bretscher, A. (1995) Ezrin self-association involves binding of an N-terminal domain to a normally masked C-terminal domain that includes the F-actin binding site. Mol. Biol. Cell 6, 1061-1075.

[5] McCartney, B. M. \& Fehon, R. G. (1996) Distinct cellular and subcellular patterns of expression imply distinct functions for the Drosophila homologues of Moesin and the neurofibromatosis 2 tumor suppressor, Merlin. J. Cell Biol. 133, 843-852.

[6] Yang, H. S. \& Hinds, P. W. (2003) Increased Ezrin expression and activation by CDK5 coincident with acquisition of the senescent phenotype. Mol. Cell 11, 1163-1176.

[7] Pearson, M., Reczek, D., Bretscher, A. \& Karplus, P. (2000) Structure of the ERM protein moesin reveals the FERM domain fold masked by an extended actin binding tail domain. Cell 101, 259-270.

[8] Krieg, J. \& Hunter, T. (1992) Identification of the two major epidermal growth factor-induced tyrosine phosphorylation sites in the microvillar core protein ezrin. J. Biol. Chem. 267, 19258-19265.

[9] Yonemura, S. et al. (1998) Ezrin/radixin/moesin (ERM) proteins bind to a positively charged amino acid cluster in the juxtamembrane cytoplasmic domain of CD44, CD43, and ICAM-2. J. Cell Biol. 140, 885-895.

[10] Weinman, E. J., Hall, R. A., Friedman, P. A., Liu-Chen, L. Y. \& Shenolikar, S. (2006) The association of NHERF adaptor proteins with G protein-coupled receptors and receptor tyrosine kinases. Annu. Rev. Physiol. 68, 491-505.

[11] Shcherbina, A., Bretscher, A., Kenney, D.M., Remold-O'Donnell, E., (1999). Moesin, the major ERM protein of lymphocytes and platelets, differs from ezrin in its insensitivity to calpain. FEBS Lett. 443, 31-36.

[12] Berryman, M., Franck, Z., Bretscher, A., (1993). Ezrin is concentrated in the apical microvilli of a wide variety of epithelial cells whereas moesin is found primarily in endothelial cells. J. Cell Sci. 105, 1025-1043

[13] Tsukita, S., Hieda, Y., (1989). A new 82-kD barbed end-capping protein (radixin) localized in the cell- to-cell adherens junction: purification and characterization. J. Cell Biol. 108, 2369-2382.

[14] Funayama, N., Nagafuchi, A., Sato, N., Tsukita, S., (1991). Radixin is a novel member of the band 4.1 family. J. Cell Biol. 115, 1039-1048.

[15] Amieva, M.R., Wilgenbus, K.K., Furthmayr, H., (1994). Radixin is a component of hepatocyte microvilli in situ. Exp. Cell Res. 210, 140-144.

[16] Franck, Z., Gary, R., Bretscher, A., (1993). Moesin, like ezrin, colocalizes with actin in the cortical cytoskeleton in cultu red cells, but its expression is more variable. J. Cell Sci. 105, 219-231.

[17] Amieva, M.R., Furthmayr, H., (1995). Subcellular localization of moesin in dynamic filopodia, retraction fibers, and other structures involved in substrate exploration, attachment, and cell-cell contacts. Exp. Cell Res. 219, 180-196.

[18] Bretscher, A., Reczek, D., Berryman, M., (1997). Ezrin: a protein requiring conformational activation to link microfilaments to the plasma membrane in the assembly of cell surface structures. J. Cell Sci. 110, 3011-3018.

[19] Bretscher, A., (1989). Rapid phosphorylation and reorganization of ezrin and spectrin accompany morphological changes induced in A-431 cells by epidermal growth factor. J. Cell Biol. 108, 921-930.

[20] Hanzel, D.K., Urushidani, T., Usinger, W.R., Smolka, A., Forte, J.G., (1989). Immunological localization of an 80-kDa phosphoprotein to the apical membrane of gastric parietal cells. Am. J. Physiol. 256, G1082-G1089. 
[21] Chen, J., Doctor, R.B., Mandel, L.J., (1994). Cytoskeletal dissociation of ezrin during renal anoxia: role in microvillar injury. Am. J. Physiol. 267, C784-C795.

[22] Takeuchi, K., Sato, N., Kasahara, H., Funayama, N., Nagafuchi, A., Yonemura, S., Tsukita, S., (1994b). Perturbation of cell adhesion and microvilli formation by antisense oligonucleotides to ERM family members. J. Cell Biol. 125, 1371-1384.

[23] Bonilha, V.L., Finnemann, S.C., Rodriguez-Boulan, E., (1999). Ezrin promotes morphogenesis of apical microvilli and basal infoldings in retinal pigment epithelium. J. Cell Biol. 147, 1533-1548.

[24] Martin, M., Andreoli, C., Sahuquet, A., Montcourrier, P., Algrain, M., Mangeat, P., (1995). Ezrin NH2-terminal domain inhibits the cell extension activity of the COOH-terminal domain. J. Cell Biol. 128, 1081-1093

[25] Cao, T.T., Deacon, H.W., Reczek, D., Bretscher, A., von Zastrow, M., (1999). Akinase-regulated PDZ-domain interaction controls endocytic sorting of the beta2-adrenergic receptor. Nature 401, 286-290.

[26] Defacque, H., Egeberg, M., Habermann, A., Diakonova, M., Roy, C., Mangeat, P., Voelter, W., Marriott, G., Pfannstiel, J., Faulstich, H., Griffiths, G., (2000). Involvement of ezrin/moesin in de novo actin assembly on phagosomal membranes. EMBO J. 19, 199-212.

[27] Louvet, S., Aghion, J., Santa-Maria, A., Mangeat, P., Maro, B., (1996). Ezrin becomes restricted to outer cells following asymmetrical division in the preimplantation mouse embryo. Dev. Biol. 177, 568-579.

[28] Polesello, C., Delon, I., Valenti, P., Ferrer, P. \& Payre, F. D (2002) Moesin controls actin-based cell shape and polarity during Drosophila melanogaster oogenesis. Nature Cell Biol. 4, 782-789.

[29] Jankovics, F., Sinka, R., Lukacsovich, T. \& Erdelyi, M. (2002) Moesin crosslinks actin and cell membrane in Drosophila oocytes and is required for OSKAR anchoring. Curr. Biol. 12, 2060-2065.

[30] Kunda, P., Pelling, A. E., Liu, T. \& Baum, B. (2008) Moesin controls cortical rigidity, cell rounding, and spindle morphogene sis during mitosis. Curr. Biol. 18, 91-101.

[31] Carreno, S. et al. (2008) Moesin and its activating kinase Slik are required for cortical stability and microtubule organization in mitotic cells. J. Cell Biol. 180, 739-746.

[32] Maddox, A. S. \& Burridge, K. (2003) RhoA is required for cortical retraction and rigidity during mitotic cell rounding. J. Cell Biol. $160,255-265$.

[33] Charras, G. T., Hu, C. K., Coughlin, M. \& Mitchison, T. J. (2006) Reassembly of contractile actin cortex in cell blebs. J. Cell Biol. $175,477-490$.

[34] Formstecher, E. et al. (2005) Protein interaction mapping: a Drosophila case study. Genome Res. 15, 376 -384.

[35] Pilot, F., Philippe, J. M., Lemmers, C. \& Lecuit, T. (2006) Spatial control of actin organization at adherens junctions by a synaptotagmin-like protein Btsz. Nature 442, 580-584.

[36] Speck, O., Hughes, S. C., Noren, N. K., Kulikauskas, R. M. \& Fehon, R. G. (2003) Moesin functions antagonistically to the Rho pathway to maintain epithelial integrity. Nature 421,83-87.

[37] Molnar, C. \& de Celis, J. F. (2006) Independent roles of Drosophila Moesin in imaginal disc morphogenesis and hedgehog signalling. Mech. Dev. 123, 337-351.

[38] Serano, J. \& Rubin, G. M. (2003) The Drosophila synaptotagmin-like protein bitesize is required for growth and has mRNA localization sequences within its open reading frame. Proc. Natl Acad. Sci. USA 100, 13368-13373

[39] Gobel, V., Barrett, P. L., Hall, D. H. \& Fleming, J. T. (2004) Lumen morphogenesis in C. elegans requires the membranecytoskeleton linker erm-1. Dev. Cell 6, 865-873.

[40] Van Furden, D., Johnson, K., Segbert, C. \& Bossinger, O. (2004) The C. elegans ezrin-radixin-moesin protein ERM-1 is necessary for apical junction remodelling and tubulogenesis in the intestine. Dev. Biol. 272, 262-276.

[41] Bossinger, O., Klebes, A., Segbert, C., Theres, C. \& Knust, E. (2001) Zonula adherens formation in Caenorhabditis elegans requires dlg-1, the homologue of the Drosophila gene discs large. Dev. Biol. 230, 29-42.

[42] Hunter, K. W. (2004) Ezrin, a key component in tumor metastasis. Trends Mol. Med. 10, 201-204.

[43] Ren, L. et al. (2009) The actin-cytoskeleton linker protein ezrin is regulated during osteosarcoma metastasis by PKC. Oncogene 28, $792-802$.

[44] Jeanes, A., Gottardi, C. J. \& Yap, A. S. (2008) Cadherins and cancer: how does cadherin dysfunction promote tumor progression? Oncogene 27, 6920-6929.

[45] Ponta, H., Sherman, L. \& Herrlich, P. A. (2003) CD44: from adhesion molecules to signalling regulators. Nature Rev. Mol. Cell Biol. 4, 33-45.

[46] Lee, J. H. et al. (2004) Roles of p-ERM and Rho-ROCK signaling in lymphocyte polarity and uropod formation. J. Cell Biol. 167, $327-337$.

[47] Yoshinaga-Ohara, N., Takahashi, A., Uchiyama, T. \& Sasada, M. (2002) Spatiotemporal regulation of moesin phosphorylation and rear release by Rho and serine/threonine phosphatase during neutrophil migration. Exp. Cell Res. 278, 112-122.

[48] Faure, S. et al. (2004) ERM proteins regulate cytoskeleton relaxation promoting T cell-APC conjugation.Nature Immunol. 5, 272279

[49] Delon, J., Kaibuchi, K. \& Germain, R. N. (2001) Exclusion of CD43 from the immunological synapse is mediated by phosphorylation-regulated relocation of the cytoskeletal adaptor moesin. Immunity 15, 691-701.

[50] Allenspach, E. J. et al. (2001) ERM-dependent movement of CD43 defines a novel protein complex distal to the immunological synapse. Immunity 15, 739-750.

[51] Roumier, A. et al. (2001) The membrane-microfilament linker ezrin is involved in the formation of the immunological synapse and in $\mathrm{T}$ cell activation. Immunity 15, 715-728.

[52] Liu, Y., Belkina, N. V. \& Shaw, S. (2009) HIV infection of T cells: actin-in and actin-out. Sci. Signal. 2 , pe23.

[53] Barrero-Villar, M. et al. (2009) Moesin is required for HIV-1-induced CD4-CXCR4 interaction, F-actin redistribution, membrane fusion and viral infection in lymphocytes. J. Cell Sci. 122, 103-113.

[54] Kubo, Y. et al. (2008) Ezrin, Radixin, and Moesin (ERM) proteins function as pleiotropic regulators of human immunodeficiency virus type 1 infection. Virology 375, 130-140.

[55] Doulet, N. et al. (2006) Neisseria meningitidis infection of human endothelial cells interferes with leukocyte transmigration by preventing the formation of endothelial docking structures. J. Cell Biol. 173, 627-637. 